\title{
Scheduling Flexible Flow Shops with Sequence-Dependent Setup Effect *
}

\author{
Chung-Yang Liu \\ Department of Electrical Engineering \\ National Taiwan University \\ Taipei, Taiwan, R.O.C. \\ email:cyliu@ac.ee.ntu.edu.tw
}

\begin{abstract}
This paper presents an optimization model-based approach for production scheduling of flexible flow shops where sequence-dependent setup effect is significant. The scheduling problem is formulated as an integer programming problem. In the presence of setup effect, individual machine setup status must be scheduled in accordance with part processing schedule. A Lagrangian relaxation method is adopted to solve the problem in dual space, where the synchronization constraint between machine setup and part processing is relaxed. An efficient heuristic is developed to adjust the dual solution to primal feasibility. Network flow structures of both machine status change and part flow balance equations are exploited for algorithm development. Numerical results demonstrate that our methodology generates an optimal schedule on a small example and a near-optimal solution on a multi-stage and multi-machine example using a reasonable amount of CPU time.
\end{abstract}

\section{Introduction}

Scheduling with sequence-dependent setup time consideration is one of the most difficult classes of scheduling problems. When a machine switches from processing one production type to another, setup time may be incurred because of changing the accessory. The flow time of production and machine setups is influenced by production mix and production sequence. Processing in large batches may increase machine utilization and reduce setup time. But it also increases the flow time. Therefore the scheduling problems with setup time consideration are to tradeoff the work in process level between flow time and machine utilization by selecting batch size and schedule.

$A$ one-machine sequence-dependent setup scheduling problem is equivalent to a traveling-salesman problem [13] and is NP-hard. Machine setup time is a significant factor in the production scheduling of most factories; it may easily take more than $20 \%$ of machine available capacity if not well handled.

The study of scheduling problem with sequencedependent setup effects has attracted quite an amount of attention in recent years. However, most research neglected the setup times or simplified the problem by combining setup time with processing time and focused mostly on the single machine problem. Dynamic programming, integer programming, branch and bound, and enumeration methods have been used as

*This work was supported in part by the National Science Council of the Republic of China under Grants NSC84-2212E002-075 and NSC85-2212-E002-074. solution tools. Ovacik and Uzsoy presented a family of rolling horizon heuristic to minimize maximum lateness on a single machine in the presence of sequencedependent setup times [12]. Bai and Elhatsi studied a deterministic one-machine, 2-part-type setup system by using optimal feedback control frameworks [1].

Multi-machine scheduling problems are more challenging than single-machine ones because the allocation of machines adds another dimension of complexity [13]. Many heuristics and rules have been published to solve the multi-machine problems [4][17]. Vemuaganti etc. used Lagrangian decomposition and network flow structure to solve this scheduling problem but only considered the setup cost [16]. Dietrich and Escudero considered a single-stage, unrelated machines and multiple job-types by using branch \& bound method [7]. In summary, very few researchers have succeeded in solving the multi-stage, multi-machine setup time scheduling problem.

In this paper, we focus on scheduling flexible flow shops (FFS) with sequence-dependent setup effects. The flows of work are unidirectional [6]. The scheduling problem schedules the production and machine 1) to meet customers' due dates in just-in-time philosophy, 2) to reduce work in process (WIP), and 3) to reduce the machine setup times, while satisfying the system constraints such as machine capacity, end product demands, and precedence relationship.

As we know, production scheduling determines when to process what and where the goal is met and machine scheduling determines the jobs sequence of a machine. In a feasible schedule, the production scheduling must synchronize with machine scheduling, as shown in Figure 1.1.

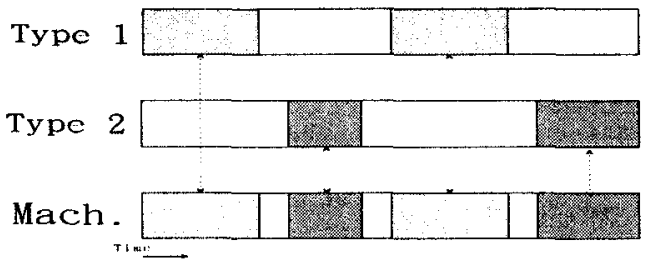

Figure 1.1: Synchronization of Production And Machine Scheduling

We formulate this scheduling problem into a largescale integer linear programming problem which is NP-hard in computational complexity [13]. Instead of finding the optimal schedule of this NP hard-problem, we use a sub-optimal approach. A Lagrangian relaxation and network flow-based approach is develope- 
d by extending Chang and Liao's method for scheduling flow shops of no setup effects[6]. We use the Lagrangian relaxation method to relax the synchronization constraints and then decompose this problem into production scheduling subproblems and machine scheduling subproblems. The minimum cost linear network flow (MCLNF) model is applied to production scheduling subproblems as in [6]. In this paper we also apply MCLNF to machine scheduling subproblems with setup consideration. To find good Lagrangian multipliers, we simplify Tomastik and Luh's method [14] to find the searching direction. We then develop an efficient heuristic to adjust the relaxed solution to a feasible one by exploiting the network structure. Preliminary numerical experiments are conducted to examine the feasibility and optimality of our algorithm. Results demonstrate that our algorithm can obtain good solutions in reasonable amount of CPU times.

The remainder of this paper is organized as follows. In Section 2, we formulate the scheduling problem mathematically. The solution methodology is described in Section 3. Numerical results are demonstrated and analyzed in Section 4 . Section 5 concludes this paper.

\section{Problem Formulation}

Consider a make-to-order flexible flow shop where machine setup times are significant as compared to the processing time. We assume that (1) setup times dependent on the job-processing sequence, (2) production flow of all types of parts go through all stages in the same sequence, (3) the processing of each stage is performed by a unique machine group, (4) there are a limited number of identical machines in each machine group, (5) each order requests only one type of products with a given due date and all demands are released at the beginning of the scheduling horizon, (6) machine failures are not considered, (7) one machine can only process one product at a time, (8) raw materials are in good supply, and (9) buffer space availability does not pose a significant constraint.

To formulate the scheduling problem of this FFS, let us define the following notations :

Input Variables:

$T$ : the time horizon of scheduling;

I: total number of product types;

$\mathrm{J}$ : total number of processing stages;

$M_{j}$ : the set of machines which can process the production in stage- $j, j=1, \ldots, \mathrm{J}$

$\mathrm{K}$ : total number of orders;

$E_{k}:$ the desired product type of order-k, $\mathrm{k}=1, \ldots, \mathrm{K}$;

$D_{k}$ : the desired quantity of type- $E_{k}$ in order-k;

$a_{k}$ : the earliness penalty coefficient of order- $k$;

$b_{k}$ : the tardiness penalty coefficient of order- $k$;

$h_{k b}$ : the holding cost per unit of order- $k$ in buffer b;

$d_{k}$ : due date of order $\mathbf{k}$;

$C_{l i j}$ : cost of setting up a machine in group $M_{j}$ from processing type- $l$ to processing type- $i$

$P_{i j}$ : the time for processing a type-i product at stage$j$;

$A_{i j j}$ : time for setting up a machine in group $M_{j}$ from processing type- $l$ to processing type- $i$

Intermediate Variables:

$\zeta_{i t}^{m}: \zeta_{i t}^{m}=1$, if machine $m$ is idle but ready to process type- $i$ products at time $t ; \zeta_{i t}^{m}=0$, otherwise;
$B_{k j t}$ : the input buffer level of order- $k$ products at stage$j$ by the beginning of time period $t$ with $B_{k 11}=$ $D_{k}$, for all $\mathrm{k}=1, \ldots, \mathrm{K}$ and $\mathrm{t}=1, \ldots, \mathrm{T}$.

Decision Variables:

$u_{k j t}^{m}: u_{k j t}^{m}=1$, if one order- $k$ product is loaded for processing stage- $j$ by machine- $m$ at the beginning of time period $t ; u_{k j t}=0$, otherwise;

$\alpha_{i t}^{m}: \alpha_{i t}^{m}=1$, if machine $m$ starts to process a type- $i$ product at time $t ; \alpha_{i t}^{m}=0$, otherwise;

$\xi_{i i t}^{m}: \xi_{l i t}^{m}=1$, if machine $m$ starts to setup from processing type- $l$ to type- $i$ for stage- $j$ at time $t$; $\xi_{\text {lit }}^{m}=0$, otherwise;

In the flow line, a part of order $\mathrm{k}$ (type $E_{k}$ ) loaded onto a machine $m$ for processing stage $\mathrm{j}-1$ at time $t$ $P_{E_{k}(j-1)}+1$ goes into buffer $\mathrm{j}$ after $P_{E_{h}(j-1)}$ periods of processing. The production flows for an order in the line can therefore be described by

Flow Balance Equations:

$$
\begin{aligned}
B_{k 11} & =D_{k} \\
B_{k 1(t+1)} & =B_{k 1 t}-\sum_{m \in M_{1}} u_{k 1 t}^{m} \\
B_{k j(t+1)} & =B_{k j t}-\sum_{m \in M_{j}} u_{k j t}^{m} \\
& +\sum_{m \in M_{j-1}} u_{k(j-1)\left(t-P_{\mathbb{B}_{k}(j-1)}^{m}+1\right)}^{m} \\
B_{k(J+1)(t+1)} & =B_{k(J+1) t} \\
& +\sum_{m \in M_{J}} u_{k J\left(t-P_{\boldsymbol{B}_{k}} j+1\right)}^{m}
\end{aligned}
$$

The precedence relationship among operations of each part are implied in the flow balance equations.

It is obvious that the scheduled processing requirements must not exceed the available capacity of each machine, i.e.

Capacity Constraints:

$$
\sum_{i=1}^{I} \sum_{k=1, E_{k}=i}^{K} \sum_{\tau=t-P_{i j}+1}^{t} u_{k j \tau}^{m} \leq 1, m=1, \ldots, M .
$$

If a machine $\mathrm{m} \in M_{j}$ starts to process a type- $i$ product at time $t-P_{i j}$, is idle but ready to process a type- $i$ product at time $t-1$, or starts to setup for processing type- $i$ products from being for type- $l$ at time $t-A_{i j j}$, then machine $m$ becomes available for processing type-i products at time $t$. At time $t$, machine $m$ can either start to process type- $i$ products, be set up to processing other types, or stay idle. Note that a machine can only be used for one thing at a time. The above relation can be expressed by Machine Usage Balance Equation:

$$
\begin{aligned}
& \alpha_{i\left(t-P_{i j}\right)}^{m}+\zeta_{i(t-1)}^{m}+\sum_{l=1, l \neq i}^{I} \xi_{i\left(t-A_{l i j}\right)}^{m} \\
& =\alpha_{i t}^{m}+\zeta_{i t}^{m}+\sum_{l=1, l \neq i}^{I} \xi_{i l t}^{m}, \quad \forall m, i, t
\end{aligned}
$$

with 


$$
\sum_{i=1}^{I}\left[\alpha_{i 0}^{m}+\zeta_{i 0}^{m}+\sum_{l=1, l \neq i}^{I} \xi_{l i 0}^{m}\right]=1
$$

At last, part loading decisions must be synchronized with the machine processing decisions:

Synchronization Constraints:

$$
\sum_{k=1, E_{k}=i}^{K} u_{k j t}^{m}=\alpha_{i t}^{m}, \quad m \in M_{j}, \forall j .
$$

Note that (5) and (8) imply that $\alpha_{i t}^{m} \leq 1$ and $\sum_{i=1}^{I} \alpha_{i t}^{m} \leq 1$.

One of the objectives of scheduling is to meet customers' due dates if possible. For this purpose, we set a penalty function for both early and late deliveries. We set an interval of $w$ time units before the due date whitin which there is no penalty. The earliness and lateness penalty coefficients for each unit of order-k completed at time $t$ are defined by

$$
\begin{cases}a_{k}\left(d_{k}-w-t\right), & \text { if } t \leq d_{k}-w ; \\ 0, & \text { if } d_{k}-w \leq t<d_{k} \\ b_{k}\left(t-d_{k}\right), & \text { if } t>d_{k} .\end{cases}
$$

Coefficients $a_{k}$ and $b_{k}$ can be different among orders to reflect their relative importance. The penalty cost of one order is formulated as follow:

$$
\begin{aligned}
v_{k} & =a_{k} \sum_{t=1}^{d_{k}-w} \sum_{m \in M_{J}}\left(d_{k}-w-t\right) u_{k J t}^{m} \\
& +b_{k} \sum_{t=d_{b}+1}^{T} \sum_{m \in M_{J}}\left(t-d_{k}\right) u_{k J t}^{m}, \forall k .
\end{aligned}
$$

Other objectives of scheduling include the reduction of work in-process and setup costs [5]. The production scheduling problem is then to determine a schedule that minimizes the total production cost while satisfying all the system constraints. The scheduling problem $(P)$ can now be formulated as

$$
\begin{aligned}
& \text { (P) } \min _{\iota, \alpha, \xi} \sum_{i=1}^{I} \sum_{k=1, E_{k}=i}^{K}\left(v_{k}\right. \\
& \left.+\sum_{t=1}^{T} \sum_{b=1}^{J} h_{k b} B_{k b t}\right) \\
& +\sum_{j=1}^{J} \sum_{m \in M_{j}} \sum_{i=1}^{I} \sum_{l=1, l \neq i}^{I} \sum_{t=1}^{T} C_{l i j} \xi_{l i t}^{m}
\end{aligned}
$$

subject to constraints $(1) \sim(8)$,

where the first term is incurred by penalty for early and tardy deliveries, the second by holding in-process inventories and the third by machine setups.

\section{Lagrangian Relaxation and Net- work Flow Approach}

Decision variables in $(P)$ can clearly be divided into two groups: $\left\{u_{k j t}^{m}\right\}$ for production schedule of parts and $\left\{\alpha_{i t}^{m}, \xi_{i t}^{m}\right\}$ for machine usage schedule. The coupling between these two groups is through synchronization constraint (8). If constraint (8) did not exist, problem (P) would essentially consist of two classes of independent scheduling subproblems. The first class would be production scheduling subproblems(PSS) for individual orders, which minimized the sum of delivery penalty cost and the in-process inventory cost subject to constraints $(1) \sim(5)$. The second class would be individual machine scheduling subproblem (MSS) which minimized the setup cost subject to constraints (6) and (7). The part flow balance equations (1) (4) and the machine usage balance equation (6) render themselves to a network structure. Intuitively, when all the machines satisfy their respective machine usage balance equations and the production scheduling of parts are synchronized with machine schedules, there are then no capacity violation, i.e., constraints $(6),(7)$ and (8) imply (5).

Motivated by the above observations, we adopt a solution approach based on Lagrange relaxation and network flow optimization[6]. After relaxing the synchronization constraints $(8)$, the problem $(P)$ could be decomposed into independent production and machine scheduling subproblems, and have a network structured constraint set each.

Lagrangian relaxation can relax the complicating constraints using Lagrangian multipliers and decouples the decision variables. As a result of relaxing the constraints, the Lagrangian dual function is formed which is a function of the lagrangian multiplies. The subgradient method is frequently used as a method for updating the Lagrangian multipliers. To improve the convergence and computational efficiency of subgradient method, Tomastik and Luh [14] have presented a facet ascend method which can search for an optimizing direction and move along this direction to find a set of good Lagrangian multipliers. To reduce the algorithm complexity, we simplify the direction searching method of [14]. The optimizing searching direction of our method is the intersection of two adjacent facets.

Although it can be shown that the dual problem is concave, the subgradient algorithm may be stopped before the dual optimum is found because of limitation on available computation time. In such case, heuristics can be used to get a near-optimal, feasible solution of $(\mathrm{P})$ based on the infeasible solution obtained from solving; the dual problem. We also develop a feasibility adjustment heuristic that accounts for the consideration of machine setup. The relaxation results of easier-to-solve subproblems provide a lower bound of the optimal solution to the original problem. The feasibility adjustment heuristic generates an upper bound of the optimal solution. The gap between the two bounds is called duality gap which indicates the optimality of the feasible schedule.

III.1 Decomposition by Lagrangian Relaxation

Applying Lagrangian relaxation to the synchronization constraints, we form a Lagrangian function for (P) as

$$
\begin{aligned}
& \sum_{i=1}^{I} \sum_{k=1, E_{k}=i}^{K}\left(v_{k}+\sum_{t=1}^{T} \sum_{b=1}^{J} h_{k b} B_{k b t}\right) \\
+ & \sum_{j=1}^{J} \sum_{m \in M_{j}} \sum_{i=1}^{I} \sum_{l=1, l \neq i}^{I} \sum_{t=1}^{T} C_{l i j} \xi_{l i t}^{m}
\end{aligned}
$$




$$
+\sum_{j=1}^{J} \sum_{m \in M_{j}} \sum_{i=1}^{I} \sum_{t=1}^{T} \pi_{i m t}\left(\alpha_{i t}^{m}-\sum_{k=1, E_{k}=i}^{K} u_{k j t}^{m}\right)
$$

where $\left\{\pi_{i m t}\right\}$ are Lagrange multipliers.

The function above is separable in $\boldsymbol{u}_{k}, \boldsymbol{\alpha}_{m}$ and $\boldsymbol{\xi}_{m}$, where $\boldsymbol{u}_{k} \equiv\left\{u_{k j t}^{m}, \forall j, t, m\right\}$, where $\boldsymbol{\alpha}_{m} \equiv\left\{\alpha_{i t}^{m}, \forall i, t\right\}$ and $\boldsymbol{\xi}_{m} \equiv\left\{\xi_{i t}^{m}, \forall i, t\right\}$. For a given set of Lagrange multipliers $\pi \equiv\left\{\pi_{i m t}, \forall i, m, t\right\}$, we define for the required parts of order-k

$$
\begin{aligned}
P S_{k}\left(u_{k}, \pi_{E_{k}}\right) & \equiv\left(v_{k}+\sum_{t=1}^{T} \sum_{b=1}^{M} h_{k b} B_{k b t}\right) \\
& -\sum_{t=1}^{T} \sum_{j=1}^{J} \sum_{m \in M_{j}} \pi_{E_{k} m t} u_{k j t}^{m}
\end{aligned}
$$

where $\pi_{E_{k}} \equiv\left\{\pi_{E_{k} m t}, \forall m, t\right\}$, and for machine $m$

$$
\begin{aligned}
M S_{m}(\xi, \alpha, \pi) & \equiv \sum_{t=1}^{T}\left(\sum_{i=1}^{I} \sum_{i=1, l \neq i}^{I} C_{l i j} \xi_{i i t}^{m}\right. \\
& \left.+\sum_{i=1}^{I} \pi_{i m t} \alpha_{i t}^{m}\right) .
\end{aligned}
$$

The dual problem to $(P)$ after Lagrangian relaxation can then be defined in the following form

$$
\begin{aligned}
&(D) \max _{\pi}\left\{\Phi(\pi) \equiv \sum_{i=1}^{I} \sum_{k=1, E_{k}=i}^{K} \min _{u_{k}} P S_{k}\left(u_{k}, \pi\right)\right. \\
&\left.+\sum_{j=1}^{J} \sum_{m \in M_{j}} \min _{\xi_{m}, \alpha_{m}} M S_{m}\left(\xi_{m}, \alpha_{m}, \pi\right)\right\} \\
& \text { subject to (1) (7). }
\end{aligned}
$$

For a given set of Lagrangian multipliers $\pi$ (i.e. after relaxing the synchronization constraints), there are two classes of independent subproblem in (D):

1) production scheduling subproblem for the required parts of order-k, for $k=1, \ldots, K$,

$$
(P S-k) \quad \sum_{i=1}^{I} \sum_{k=1, E_{k}=i}^{K} \min _{u_{k}} P S_{k}\left(u_{k}, \pi\right)
$$

subject to constraints (1) (5);

2) machine scheduling subproblem for machine $m$,

$$
(M S-m) \quad \sum_{j=1}^{J} \sum_{m \in M_{j}} \min _{\xi_{m}, \alpha_{m}} M S_{m}\left(\xi_{m}, \alpha_{m}, \pi\right)
$$

subject to constraints $(6) \sim(7)$.

\section{III.2 Network Flow Algorithm}

A. Production Scheduling Subproblem

Each (PS-k) can be formulated as a minimum cost linear network flow (MCLNF) problem due to the network structure of the flow balance equations. Interested reader may refer to [6] for more detail.

\section{B. Machine Scheduling Subproblem}

The machine usage balance equations (6) of (MSm) can also be formulated as a MCLNF problem. We could construct a network representation for constraint (6) of each machine. Figure 3.1 illustrated the network representation of a 2-type, 6-period example, where the processing time for type-1 is 3 units, and setup time is 2 units for setting up form type-1 to type2 . The horizontal arcs correspond to making machine idle with cost as zero, and each lasts for one time interval. The curved arcs represent machine processing and the arc cost is the appropriate Lagrangian multiplier in (MS-m). The others are machine setup with it appropriate arc cost. We adopt the RELAX code, which is developed by Bertsekas and Tseng, to solve these MCLNF problems [2].

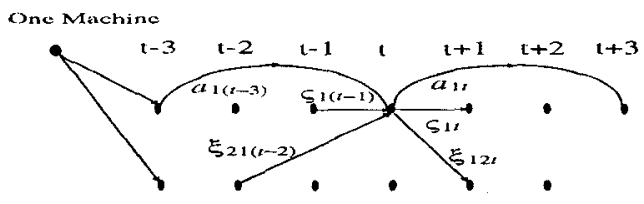

Figure 3.1: Network Flow Representation of Machine Usage Balance Equation

III.3 Lagrangian Multipliers Adjustment After solving all the subproblems for a given set of Lagrangian multipliers $(\pi)$, the dual function $\Phi$ $(\pi)$ can be evaluated. Since the dual function is polyhedral concave, is made up of many facets and is nondifferentiable [14], we iteratively update $(\pi)$ by modifying a facet ascending method of [14].

We have observed that two adjacent facets of dual function $\Phi(\pi)$ must form an intersection which often provides a good searching direction. Our modification is designed to reduce the algorithmic complexity of finding a good searching direction in [14]. Our method finds the intersection of two adjacent facets and takes this intersection as the searching direction.

\section{III.4 Constructing a Good Feasible Schedule}

The primal problem (P) is not a convex optimization problem because of the integer decision variables. The dual solution may be infeasible, i.e, some of the synchronization constraints may be violated. We develop an iterative algorithm that exploits the network structures of material flows and machine usage to adjust the dual solution to a near-optimal feasible schedule.

The heuristic first checks all production scheduling subproblems and machine scheduling subproblems over all time periods to identify whether the synchronization constraints are satisfied or not. We then retain the parts of the schedule which satisfy the synchronization constraints. We then design a grouping algorithm that groups temporality adjacent processing of the same type on a machine into a batch to increase machine utilization.

CGFSA

step 0: Initialize with the dual problem solution.

step 1: Identify the feasible parts of production schedules according to machine schedules.

Do for $m$ from 1 to $M$

Do for i from 1 to I 
Do for $t$ from 1 to $T$

Check if the synchronization constraint of machine- $m$ and product type- $i$ is not violated at time period-t. If so, retain the feasible schedule; if not, discard the infeasible part of the schedule. Enddo

\section{Enddo}

Enddo

step 2: Perform grouping of the feasible part of schedule identified by step 1 by the grouping algorithm described in the Appendix.

step 3: Identify orders which are scheduled but the scheduled quantities are insufficient. Select from them an order with the earliest due date.

step 4: Find the unscheduled machine times from machine schedules and construct the production network of the selected order, where the paths are the available machine-times of all needed machine. Reschedule the insufficient amount of the selected order by solving a MCLNF for this order. Then perform grouping of these rescheduled parts into the existing schedule.

step 5: If there are still orders with insufficient parts to be rescheduled, goto step3; else end.

\section{Numerical Results}

Numerical experimentation results are presented in this Section to demonstrate the feasibility and optimality of our algorithms. We first apply our algorithm to a multi-machine, one-stage and two-product example, where an optimal schedule can be obtained by observation and some characteristics of our algorithm can be examined. We then consider a multistage and multi-product example. In this example, we demonstrate that our algorithm could reduce the setup times and delivery penalty. We also make a comparison with a scheduling method [10] which combines setup time into processing time.

Our algorithm is implemented in $\mathrm{C}$ code and all of our experimentations are performed on a PC-486. The processing times and setup times of all stages for examples 1 and 2 are listed in Tables 4.1 and 4.2 respectively.

Table 4.1 Processing Time
\begin{tabular}{|c||c|c|}
\hline Product & Stage1 & StageZ \\
\hline $\mathrm{A}$ & 1 & 1 \\
\hline $\mathrm{B}$ & 1 & 1 \\
\hline Table 4.2 Setup Time
\end{tabular}
\begin{tabular}{|c||c|}
\hline Setup & Setup Time \\
\hline $\mathrm{A} \rightarrow \mathrm{B}$ & \multicolumn{2}{|c|}{2} \\
\hline$\overline{\mathrm{B} \rightarrow \mathrm{A}}$ & 2 \\
\hline
\end{tabular}

We set cost function coefficients $a_{k}=1, b_{k}=10 \forall k$, $h_{k b}=1 \forall k, b$ and $C_{l i j}$ (setup cost) $=10 \forall l, i, j$. All the multipliers are initialized as zero.

\section{Example 1: A Simple Example}

This is a two-machine, single-stage example which schedules two orders over ten time periods. Order information is given in Table 4.3. The schedule obtained by using our algorithm is shown in Figure 4.1.

Table 4.3 Orders of Example1

\begin{tabular}{|c||c|c|c|}
\hline Order-No & Product & Quantity & Due Date \\
\hline 1 & A & 6 & 5 \\
\hline 2 & B & 6 & 10 \\
\hline
\end{tabular}

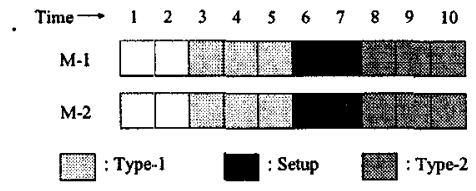

Figure 4.1: Schedule of Example1

In this example, order-1's due date is earlier than order-2's. The optimal schedule sets both machines M-1 and M-2 to process order 1 products starting from time unit 3 , and then sets them up to process order-2's products. The resultant duality gap of our algorithm for this example is $0 \%$ and CPU time spent is 1.16 seconds.

\section{Example 2: Multi-Machine Multi-Stage Example}

In this example, there are two product orders of the same due date, each requiring two processing stages. There are two homogeneous machines for processing each stage. Intuitively, the optimal schedule of this example is to divide machines equally to process the products of order-1 and 2. The result of our scheduling algorithm is shown in Figure 4.2.

Table 4.4 Orders of Example2

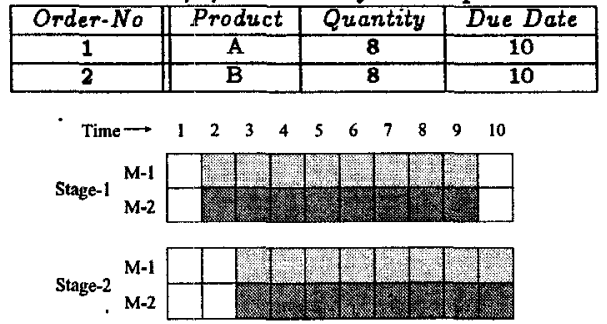

Figure 4.2: Schedule of Example2

Solution to this example by our algorithm is as expected, where no setup times are incurred in the schedule and all products meet due date. The duality gap is $1.8375 \%$ and CPU time spent is 2.53 seconds.

Now, based on the same production data, we use another scheduling system [10] which combines the processing time and setup time to make a comparison. In this example, we assume that setup time takes $20 \%$ machine avallable capacity, i.e. the processing times of scheduling system [10] are longer than original value by $20 \%$. The schedule obtained is shown in Figure 4.3 (A). Based on the sequence of this schedule, we use the real processing times and the setup time consideration to obtain a realistic schedule shown in Figure 4.3 (B), where setup times are incurred and two units of order-2 are over-due. Clearly, the production cost of Figure $4.3(\mathrm{~B})$ is higher than ours.

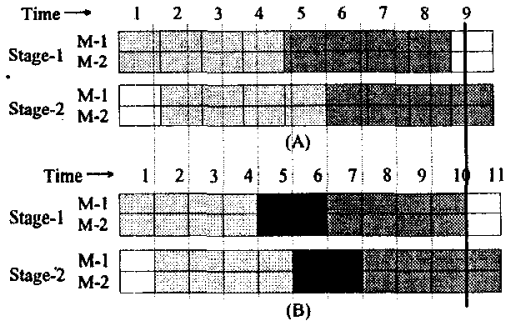

Figure 4.3: Schedule of Example2 


\section{Conclusion}

In this paper, an optimization problem formulation and solution methodology are developed for solving a flexible flow shop scheduling problem where multiple stages, multiple machines and sequence-dependent setup times are considered. The solution algorithm combines Lagrangian relaxation and minimum cost linear network flow methods to generate a nearoptimal schedule. The machine usage relationship is represented by using network structure and solved efficiently by using MCLNF algorithm. Numerical results have demonstrated the feasibility of the algorithm. Industrial applications of the proposed methodology will be reported in the future.

\section{Appendix:Grouping Algorithm}

It is easy to observe that to satisfy the precedence constraints, the processing of parts before the stage of the maximum processing time in the part's process flow $\left(j^{*}\right)$ may be grouped foreward in the schedule, and the processing of parts in the stages after $j^{*}$ must be grouped backward. In the method the schedule of the first part of stage 1 and the last part of stage-J are fixed.

step 1: set $j_{*}=\arg \max _{j}\left\{P_{i j}, \forall j\right\}$, and set $j_{0}^{+}=$

$$
j_{0}^{-}=j *, n=1, m=1 \text {; }
$$

step 2: set $j_{n}^{+}=\arg \max _{j}\left\{P_{i j}, j=1, \ldots, j_{n-1}^{+}-1\right\}$;

step 3: If $j_{n}^{+} \neq 1, \mathbf{n}=\mathrm{n}+1$, goto step 2;

step 4: set $j_{m}=\arg \max _{j}\left\{P_{i j}, j=j_{m-1}+1, \ldots, J\right\}$;

step 5: If $j_{m} \neq J, \mathrm{~m}=\mathrm{m}+1$, goto step 4;

step 6: Do from $j_{n}^{+}$to $j_{1}^{+}$

The first part of stage $j_{n}^{+}$are fixed, and the other parts of this stage are grouped foreward.

When a part in stage $j_{n}^{+}$is scheduled to an earlier time by $k$ time periods, the processing of this part in stages between $j_{n}^{+}$and $j_{(n-1)}^{+}$can only be scheduled $\mathbf{k}$ time periods earlier. enddo;

step 7: Do from $j_{m}^{-}$to $j_{1}^{-}$

The last part of stage $j_{M}^{-}$are fixed, and the other parts of this stage are grouped backward.

When a part in stage $j_{m}^{-}$is scheduled to a later time by $k$ time periods, the processing of this part in stages between $j_{m}^{-}$and $j_{(m-1)}^{-}$can only be scheduled $\mathrm{k}$ time periods later. enddo

\section{References}

[1] S. X. Bai and M. Elhafsi, ${ }^{\text {OOptimal Feedback }}$ Control of a Manufacturing System with Setup Changes", Proceedings of the 1994 IEEE Conference on Decision and Control, pp.191-196, 1994.

[2] D. P. Bertsekas and P. Tseng, "Relaxation Methods for Minimum Cost Ordinary and Generalized Network Flow Problems," Operations Research, Vol. 36, No. 1, pp. 93-114, 1988.

[3] M. H. Burman and S. B. Gershwin, "An Algorithm for the Scheduling of Setups and Production to Meet Due Dates "Proceeding of the 1992 IEEE International Conference on Robotics and Automation, pp. 1150-1156, Nice, France, May 1992.
[4] J. Cao and D. D. Bedworth, "Flow Shop Scheduling in Serial Multi-product Processes with Transfer and Setup Times",INT. J. PROD. RES., 1992, Vol. 30, No. 8, pp. 1819-1830.

[5] S.-C. Chang and F. S. Hsieh, "Order and Production. Scheduling/Rescheduling for Flow Shop$\mathrm{s}$ ", Proceedings of the 1992 International Conference on Robotics and Automation Nice, France, May, 1992, pp.1973-1978.

[6] S.-C. Chang and D.-Y. Liao, "Scheduling Flexible Flow Shops with No Setup Effects," IEEE Transactions on Robotics and Automation, Vol. 10, No. 2, pp.112-122, April 1994.

[7] B. L. Dietrich and L. F. Escudero, "On Solving a 0-1 Model for Workload Allocation on Parallel Unrelated Machines with Setups", Proceeding of the Third ORSA/TIMS Conference on Flexible Manufacturing System : Operations Research Models and Applications, pp. 181-186, 1989.

[8] M. L. Fisher, "Lagrangian Relaxation Method for Solving Integer Programming Problems," Management Science, Vol. 27, pp. 1-18, 1981.

[9] A. Gascon, R. C. Leachman, "A Dynamic Programming Solution to the Dynamic, Multi-Item, Single-Machine Scheduling Problem", Operation Research, Vol. 36, NO.1, January-February 1988.

[10] C.-Y. Iiu, S.-C. Chang, H.-M. Hsu, C.-C.Chen, "Scheduling for IC Testing and Assembly", $A u-$ tomation'94 Proceeding, Vol. 4, pp. 59-67, Taipei, Taiwan, R.O.C., 1994.

[11] P. B. Luh, D. J. Hoitomt, E. Max, and K. R. Pattipati, "Scheduling Generation and Reconfiguration for Parallel Machines," IEEE Trans. Robotics and Automation, Vol. 6,1 No. 6, pp. 687 696, DEC. 1990.

[12] I. M. Ovacik and R. Uzsoy, "Rolling Horizon Algorithm for a Single-Machine Dynamic Scheduling Problem with Sequence-Dependent Setup Times", INT. J. PROD. RES., 1994, Vol. 32, No. 6, pp.1243-1263.

[13] M. Pinedo, "Scheduling Theory, Algorithms, and Systems", Prentice Hall, Inc. 1995.

[14] R. Tomastik and P. B. Luh, "The Facet Ascending Algorithm for Integer Programming Problems" Proceedings of the 32nd IEEE Conference on Decision and Control, San Antonio, Texas, December 1993, pp.2880-2884.

[15] R. Uzsoy, C.-Y. Lee and L. A. Martin-Vega, "A Review of Production Planning and Scheduling Models in the Semiconductor Industry - Part I: System Characteristics, Performance Evaluation and Production Planning," IIE Transactions, Vol. 24, No. 4, pp. 47-60, September 1992.

[16] R. R. Vemuganti, H. Arsham and S. P. Shao Jr, "An Implementation of Lagrangian Decomposition in Solving a Multi-Item Production Scheduling Problem with Changeover Cost and Restrictions", Math. Comput. Modelling, Vol. 12, No.12, pp.1437-1446, 1989

[17] C. Zhou and P. J. Egbelu, "Scheduling in a Manufacturing Shop with Sequence Dependent Setups", Robotics \& Computer-Integrated Manufacturing, Vol. 5, No. 1, pp.73-81, 1989. 